\title{
ON STATISTICAL PROPERTIES OF THE EXPONENTIATED TRANSMUTED INVERTED WEIBULL DISTRIBUTION
}

\section{OGUNDE ADEBISI ADE ${ }^{1}$, OMOSIGHO DONATUS OSARETIN ${ }^{1}$ and FATOKI OLAYODE ${ }^{2}$}

\author{
${ }^{1}$ Department of Mathematics and Statistics \\ The Federal Polytechnic Ado-Ekiti \\ Nigeria \\ e-mail: debiz95@yahoo.com \\ etindon@yahoo.co.uk \\ 2Department of Statistics \\ Ogun State Institute of Technology \\ Igbesa, Ogun State \\ Nigeria \\ e-mail: fatoki_olayode@yahoo.com
}

\begin{abstract}
This work introduces a new generalization of the one parameter inverted Weibull distribution. The quadratic rank transmutation approach has been investigated. This new distribution is named exponentiated transmuted inverted Weibull (ETIW) distribution which is flexible and capable of modelling various shapes of ageing and failure characteristics. The properties of the new model are discussed and the maximum likelihood estimation is used to estimate the parameters. Explicit expressions were derived for the quantile, moment, and order statistics were examined.
\end{abstract}

2010 Mathematics Subject Classification: 70-XEX, 65EXX.

Keywords and phrases: exponentiated, transmutation map, maximum likelihood estimation, quantile.

Received November 24, 2017

(ㄷ) 2017 Scientific Advances Publishers 


\section{Introduction}

In analyzing lifetime data inverted Weibull distribution is one of the most popular probability distribution to modelling the life time data with some monotone failure rates. In [1], Flair et al. studied the properties of the inverted Weibull distribution and its application to failure data. In [17], Ogunde et al. investigates the properties of the transmuted inverted Weibull distribution. In [2], Mudholkar et al. introduced the exponentiated Weibull distribution as generalization of the standard Weibull distribution and applied the new distribution as a suitable model to the bus-motor failure time data. In [11], Khan et al. explained the flexibility of the three parameters inverted Weibull distribution and its interested properties. In [3], Mudholkar and Hutson reviewed the exponentiated Weibull distribution with new measures. In [4], Aryal and Tsokos studied the properties of transmuted Weibull distribution. In [6], Merovci proposed and studied the various structural properties of the transmuted Rayleigh distribution. In [7], Khan and King introduced the transmuted modified Weibull distribution. Transmuted Lomax distribution is presented by Ashour and Eltehiwy [8]. In [9], Merovci and Puka introduces transmuted Pareto distribution. Transmuted generalized linear exponential distribution introduced by Elbatal et al. [10]. In [18], Hady Ebraheim examined the properties of the exponentiated transmuted Weibull distribution as a generalization of the Weibull distribution.

\section{Exponentiated Transmuted Inverted Weibull (ETIW) Distribution}

A random variable $T$ is said to have a one parameter inverted Weibull distribution with parameter $\beta>0$, if its cumulative density function (cdf) is given by

$$
G(t)=e^{-t^{-\beta}} \quad t \geq 0
$$


and the probability density function is given

$$
g(t)=\beta x^{-\beta} e^{-t^{-\beta}} .
$$

A random variable $T$ is said to have a transmuted distribution if its cumulative density function (cdf), $W(t)$ is given by

$$
W(t)=(1+\lambda) G(t)-\lambda[G(x)]^{2},
$$

where $G(t)$ is the cdf of the base distribution function. The cdf, $F(t)$ of the exponentiated transmuted distribution is given by

$$
F(t)=W^{\nu}(t)=\left\{(1+\lambda) G(t)-\lambda[G(x)]^{2}\right\}^{\nu}, \quad \lambda \leq 1 .
$$

But, $f(t)=\frac{\partial F(t)}{\partial t}$ therefore differentiating Equation (4), then we have

$$
g(t)=\nu g(t)[G(t)]^{\nu-1}\{1+\lambda-2 \lambda G(t)\}\left\{1+\lambda(1-G(t)\}^{\nu-1} .\right.
$$

Combining Equations (1) and (4), give the cdf of the ETIW distribution as:

$$
F(t)=\left\{(1+\lambda) e^{-t^{-\beta}}-\lambda\left[e^{-t^{-\beta}}\right]^{2}\right\}^{\nu}
$$

Also combining Equations (1), (2), and (5), will yield the pdf of the ETIW distribution as

$$
f(t)=\nu \beta t^{-\beta}\left[1+\lambda-2 \lambda e^{-t^{-\beta}}\right]\left[e^{-t^{-\beta}}\right]^{\nu}\left[1+\lambda\left(1-e^{-t^{-\beta}}\right)\right]^{\nu-1},
$$

where $\beta>0, \nu>0$ are the shape parameters and $|\lambda|$ is the transmuted parameter. Using series representation of Prudnikov et al. [16] given as

$$
(1+x)^{\alpha}=\sum_{k=0}^{\infty} \frac{\Gamma(\alpha+1)}{\Gamma(\alpha-k+1)} \frac{x^{k}}{k !}
$$


Equation (6) we transform to

$$
f(t)=\nu \beta t^{-\beta}\left[1+\lambda-2 \lambda e^{-t^{-\beta}}\right]\left[e^{-t^{-\beta}}\right]^{\nu} \sum_{k=0}^{\infty} \frac{\Gamma(\nu)}{\Gamma(\nu-k)} \frac{\lambda^{k}}{k !}\left(1-e^{-t^{-\beta}}\right)^{k} .
$$

Using the generalized binomial expansion, given as

$$
(1-z)^{b}=\sum_{l=0}^{\infty}(-1)^{l}\left(\begin{array}{l}
b \\
l
\end{array}\right) Z^{l}
$$

Equation (9) will yield

$$
f(t)=\nu \beta t^{-\beta}\left[1+\lambda-2 \lambda e^{-t^{-\beta}}\right] \sum_{k=0}^{\infty} \sum_{l=0}^{\infty}(-1)^{l} \frac{\Gamma(\nu)}{\Gamma(\nu-k)} \frac{\lambda^{k}}{k !}\left(\begin{array}{l}
b \\
l
\end{array}\right) e^{-(l+\nu) t^{-\beta}}
$$

The graph below depicts the behaviour of the pdf of ETIW distribution at different parameters values, where $a=\nu, b=\beta$, and $c=\lambda$.
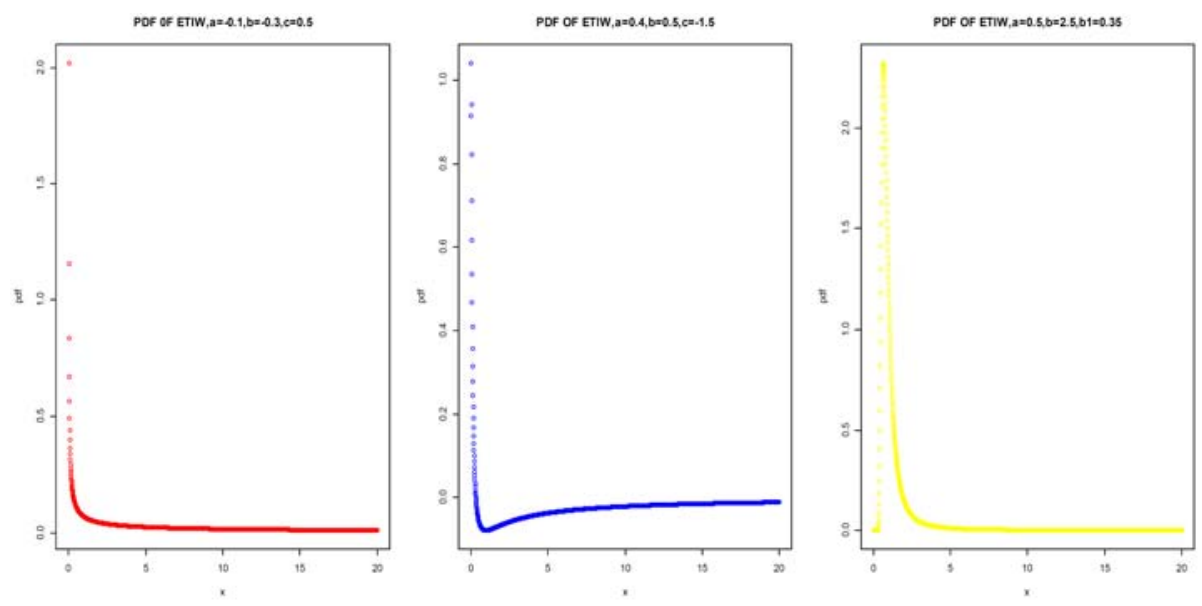

Figure 1. The graph of the pdf of ETIW distribution. 


\section{Mixture Representation of the Distribution}

Using the generalized binomial expansion given in Equation (10). We can write Equations (4) and (5) as

$$
F(t)=[G(x)]^{\nu} \sum_{i=0}^{\infty} \lambda^{i}\left(\begin{array}{l}
\nu \\
i
\end{array}\right)[1-G(x)]^{i} .
$$

Further simplification gives

$$
F(t)=[G(x)]^{\nu} \sum_{i=0}^{\infty} \sum_{j=0}^{i}(-1)^{j} \lambda^{i}\left(\begin{array}{l}
\nu \\
i
\end{array}\right)\left(\begin{array}{l}
i \\
j
\end{array}\right)[G(x)]^{i}[G(x)]^{j} .
$$

Finally, we have

$$
F(t)=\sum_{i=0}^{\infty} \sum_{j=0}^{\infty}(-1)^{j} \lambda^{i}\left(\begin{array}{l}
\nu \\
i
\end{array}\right)\left(\begin{array}{l}
i \\
j
\end{array}\right)[G(x)]^{\nu+j} .
$$

The cdf of the ETIW distribution can be expressed as a mixture density as

$$
F(t)=\sum_{i=0}^{\infty} \sum_{j=0}^{\infty}(-1)^{j} \lambda^{i}\left(\begin{array}{l}
\nu \\
i
\end{array}\right)\left(\begin{array}{l}
i \\
j
\end{array}\right) e^{-(\nu+j) t^{-\beta}}
$$

Also for the pdf we have

$$
f(t)=\nu(\nu+j) \beta t^{-\beta} \sum_{i=0}^{\infty} \sum_{j=0}^{\infty}(-1)^{j} \lambda^{i}\left(\begin{array}{l}
\nu \\
i
\end{array}\right)\left(\begin{array}{l}
i \\
j
\end{array}\right) e^{-(\nu+j) t^{-\beta}} .
$$

\section{Statistical Properties}

This section examined the statistical properties of ETIW distribution which includes the quantile, median the non-central moment, and the moment generating function. 


\subsection{Quantile function}

The $U$-th quantile function, $t_{u}$ of the ETIW distribution is the real solution of the equation

$$
F\left(t_{u}\right)=u
$$

Then, we have

$$
u=\left\{(1+\lambda) e^{-t^{-\beta}}-\lambda\left[e^{-t^{-\beta}}\right]^{2}\right\}^{\nu}
$$

On simplifying Equation (8) and also by equating $x=e^{-t^{-\beta}}$, we have

$$
u^{\frac{1}{v}}=x(1+\lambda)-\lambda x^{2}
$$

Solving the above equation using quadratic formula will yield the quantile function of ETIW given as

$$
t_{u}=-\ln \left\{\frac{1+\lambda}{2 \lambda}+\frac{\left[(1+\lambda)^{2}-4 \lambda u^{\frac{1}{\nu}}\right]^{\frac{1}{2}}}{2 \lambda}\right\}^{-\frac{1}{\beta}}
$$

The median in particular can be obtained by taking the value of $u=0.5$, then

$$
t_{0.5}=-\ln \left\{\frac{1+\lambda}{2 \lambda}+\frac{\left[(1+\lambda)^{2}-4(0.5)^{\frac{1}{\nu}} \lambda\right]^{\frac{1}{2}}}{2 \lambda}\right\}^{-\frac{1}{\beta}}
$$

An expression for the lower quartiles, upper quantile can also be obtained by taking the value of $u$ to be $0.25,0.75$, respectively. 


\subsection{Random number generation}

Using the method of inversion, random numbers from ETIW distribution can be generated with $q \sim U(0,1)$ as the solution of the equation

$$
q=\left\{(1+\lambda) e^{-t^{-\beta}}-\lambda\left[e^{-t^{-\beta}}\right]^{2}\right\}^{\nu}
$$

This gives

$$
t=-\ln \left\{\frac{1+\lambda}{2 \lambda}+\frac{\left[(1+\lambda)^{2}-4 \lambda q^{\frac{1}{v}}\right]^{\frac{1}{2}}}{2 \lambda}\right\}^{-\frac{1}{\beta}}
$$

4.3. Moments, mean, variance, skewness and kurtosis of ETIW distribution

The $r$-th order moments, for ETIW distribution can be obtained as follows for a random variable $T$ :

$$
E(T)^{r}=\int_{-\infty}^{\infty} t^{r} f(t) d t
$$

Inserting Equation (7) in (23), we have

$E(T)^{r}=\nu \beta \sum_{k=0}^{\infty} \sum_{l=0}^{\infty}(-1)^{l} \frac{\Gamma(\nu)}{\Gamma(\nu-k)} \frac{\lambda^{k}}{k !}\left({ }_{l}^{b} \int_{-\infty}^{\infty} t^{r-\beta}\left[1+\lambda-2 \lambda e^{-t^{-\beta}}\right] e^{-(l+\nu) t^{-\beta}} d t\right.$.

Expanding Equation (24) and splitting it into three, we obtain

$$
z_{1}=\nu \beta \sum_{k=0}^{\infty} \sum_{l=0}^{\infty}(-1)^{l} \frac{\Gamma(\nu)}{\Gamma(\nu-k)} \frac{\lambda^{k}}{k !}\left(\begin{array}{l}
b \\
l
\end{array}\right) \int_{-\infty}^{\infty} t^{r-\beta} e^{-(l+\nu) t^{-\beta}} d t,
$$




$$
\begin{aligned}
& z_{2}=\nu \beta \lambda t^{-\beta} \sum_{k=0}^{\infty} \sum_{l=0}^{\infty}(-1)^{l} \frac{\Gamma(\nu)}{\Gamma(\nu-k)} \frac{\lambda^{k}}{k !}\left(\begin{array}{l}
b \\
l
\end{array}\right) \int_{-\infty}^{\infty} t^{r} e^{-(l+\nu) t^{-\beta}} d t \\
& z_{3}=-2 \nu \beta \lambda t^{-\beta} \sum_{k=0}^{\infty} \sum_{l=0}^{\infty}(-1)^{l} \frac{\Gamma(\nu)}{\Gamma(\nu-k)} \frac{\lambda^{k}}{k !}\left({ }_{l}^{b} \int_{-\infty}^{\infty} t^{r} e^{-(l+\nu+1) t^{-\beta}} d t .\right.
\end{aligned}
$$

Solving for $z_{1}, z_{2}$, and $z_{3}$ by letting $u=(l+v) t^{-\beta}$ in Equations (25), (26) and $w=(l+v+1) t^{-\beta}$ in Equation (27), we have

$$
\begin{gathered}
z_{1}=-v\left(\frac{1}{l+v}\right)^{\left(1-\frac{r}{\beta}-\beta\right)} \sum_{k=0}^{\infty} \sum_{l=0}^{\infty}(-1)^{l} \frac{\Gamma(\nu)}{\Gamma(\nu-k)} \frac{\lambda^{k}}{k !}\left(\begin{array}{l}
b \\
l
\end{array}\right) \int_{-\infty}^{\infty} u^{\left(-\frac{r}{\beta}-\beta\right)} e^{-u} d u, \\
z_{2}=-\nu \lambda\left(\frac{1}{l+\nu}\right)^{\left(1-\frac{r}{\beta}-\beta\right)} \sum_{k=0}^{\infty} \sum_{l=0}^{\infty}(-1)^{l} \frac{\Gamma(\nu)}{\Gamma(\nu-k)} \frac{\lambda^{k}}{k !}\left(\begin{array}{l}
b \\
l
\end{array}\right) \int_{-\infty}^{\infty} u^{\left(-\frac{r}{\beta}-\beta\right)} e^{-u} d u, \\
z_{3}=2 \nu \lambda\left(\frac{1}{l+\nu+1}\right)^{\left(1-\frac{r}{\beta}-\beta\right)} \sum_{k=0}^{\infty} \sum_{l=0}^{\infty}(-1)^{l} \frac{\Gamma(\nu)}{\Gamma(\nu-k)} \frac{\lambda^{k}}{k !}\left(\int_{l}^{b} \int_{-\infty}^{\infty} w^{-\left(\frac{r+1}{\beta}\right)} e^{-w} d w .\right.
\end{gathered}
$$

Using a generalized gamma function to summarize Equations (28), (29) and (30) where

$$
\Gamma(\alpha)=\int_{-\infty}^{\infty} w^{\alpha-1} e^{-w} d w
$$

Then we have

$$
\begin{gathered}
z_{1}=-\nu\left(\frac{1}{l+\nu}\right)^{\left(1-\frac{r}{\beta}-\beta\right)} \sum_{k=0}^{\infty} \sum_{l=0}^{\infty}(-1)^{l} \frac{\Gamma(\nu)}{\Gamma(\nu-k)} \frac{\lambda^{k}}{k !}\left(\begin{array}{l}
b \\
l
\end{array}\right) \Gamma\left(1-\frac{r}{\beta}-\beta\right), \\
z_{2}=-\nu \lambda\left(\frac{1}{l+\nu}\right)^{\left(1-\frac{r}{\beta}-\beta\right)} \sum_{k=0}^{\infty} \sum_{l=0}^{\infty}(-1)^{l} \frac{\Gamma(\nu)}{\Gamma(\nu-k)} \frac{\lambda^{k}}{k !}\left(\begin{array}{l}
b \\
l
\end{array}\right) \Gamma\left(1-\frac{r}{\beta}-\beta\right),
\end{gathered}
$$




$$
z_{3}=2 \nu \lambda\left(\frac{1}{l+\nu+1}\right)^{\left(1-\frac{r}{\beta}-\beta\right)} \sum_{k=0}^{\infty} \sum_{l=0}^{\infty}(-1)^{l} \frac{\Gamma(\nu)}{\Gamma(\nu-k)} \frac{\lambda^{k}}{k !}\left(\begin{array}{l}
b \\
l
\end{array}\right) \Gamma\left(1-\frac{r}{\beta}-\beta\right)
$$

Then combining Equations (28), (29), and (30), we have the $r$-th moment of the ETIW distribution given as

$$
\begin{array}{r}
E(T)^{r}=\nu\left\{2 \lambda\left(\frac{1}{l+\nu+1}\right)^{\left(1-\frac{r}{\beta}-\beta\right)}-(1+\lambda)\left(\frac{1}{l+\nu}\right)^{\left(1-\frac{r}{\beta}-\beta\right)}\right\} \sum_{k=0}^{\infty} \sum_{l=0}^{\infty}(-1)^{l} \\
\times \frac{\Gamma(\nu)}{\Gamma(\nu-k)} \frac{\lambda^{k}}{k !}\left(\begin{array}{l}
b \\
l
\end{array}\right) \Gamma\left(1-\frac{r}{\beta}-\beta\right) .
\end{array}
$$

Using Equation (31), we obtain the $1^{\text {st }}, 2^{\text {nd }}, 3^{\text {rd }}$ and $4^{\text {th }}$ moment for $r=1,2,3,4$, we have

$$
\begin{aligned}
& \mu_{1}^{\prime}=\nu\left\{2 \lambda\left(\frac{1}{l+\nu+1}\right)^{\left(\frac{\beta-1-\beta^{2}}{\beta}\right)}-(1+\lambda)\left(\frac{1}{l+\nu}\right)^{\left(\frac{\beta-1-\beta^{2}}{\beta}\right)}\right\} \sum_{k=0}^{\infty} \sum_{l=0}^{\infty}(-1)^{l} \frac{\Gamma(\nu)}{\Gamma(\nu-k)} \\
& \times \frac{\lambda^{k}}{k !}\left(\begin{array}{l}
b \\
l
\end{array}\right) \Gamma\left(\frac{\beta-1-\beta^{2}}{\beta}\right), \\
& \mu_{2}^{\prime}=\nu\left\{2 \lambda\left(\frac{1}{l+\nu+1}\right)^{\left(\frac{\beta-2-\beta^{2}}{\beta}\right)}-(1+\lambda)\left(\frac{1}{l+\nu}\right)^{\left(\frac{\beta-2-\beta^{2}}{\beta}\right)}\right\} \sum_{k=0}^{\infty} \sum_{l=0}^{\infty}(-1)^{l} \frac{\Gamma(\nu)}{\Gamma(\nu-k)} \\
& \times \frac{\lambda^{k}}{k !}\left(\begin{array}{l}
b \\
l
\end{array}\right) \Gamma\left(\frac{\beta-2-\beta^{2}}{\beta}\right), \\
& \mu_{3}^{\prime}=\nu\left\{2 \lambda\left(\frac{1}{l+\nu+1}\right)^{\left(\frac{\beta-3-\beta^{2}}{\beta}\right)}-(1+\lambda)\left(\frac{1}{l+v}\right)^{\left(\frac{\beta-3-\beta^{2}}{\beta}\right)}\right\} \sum_{k=0}^{\infty} \sum_{l=0}^{\infty}(-1)^{l} \frac{\Gamma(\nu)}{\Gamma(\nu-k)} \\
& \times \frac{\lambda^{k}}{k !}\left(\begin{array}{l}
b \\
l
\end{array}\right) \Gamma\left(\frac{\beta-3-\beta^{2}}{\beta}\right)
\end{aligned}
$$




$$
\begin{array}{r}
\mu_{4}^{\prime}=\nu\left\{2 \lambda\left(\frac{1}{l+\nu+1}\right)^{\left(\frac{\beta-4-\beta^{2}}{\beta}\right)}-(1+\lambda)\left(\frac{1}{l+\nu}\right)^{\left(\frac{\beta-4-\beta^{2}}{\beta}\right)}\right\} \sum_{k=0}^{\infty} \sum_{l=0}^{\infty}(-1)^{l} \frac{\Gamma(\nu)}{\Gamma(\nu-k)} \\
\times \frac{\lambda^{k}}{k !}\left(\begin{array}{l}
b \\
l
\end{array}\right) \Gamma\left(\frac{\beta-4-\beta^{2}}{\beta}\right)
\end{array}
$$

The mean of ETIW distribution is the first moment about the origin $\left(\mu_{1}^{\prime}\right)$ which corresponds to Equation (32). It then follows that the variance $\left(\mu_{2}\right)$, the coefficient of variation $(\rho)$, the coefficient of skewness $\left(\gamma_{1}\right)$, and the coefficient of kurtosis $\left(\gamma_{2}\right)$ of ETIW distribution are respectively, obtained as

$$
\begin{gathered}
\mu_{2}=\mu_{2}^{\prime}-\left(\mu_{1}^{\prime}\right)^{2}, \\
\rho=\frac{\sqrt{\mu_{2}}}{\mu_{1}^{\prime}}=\frac{\sqrt{\mu_{2}^{\prime}-\left(\mu_{1}^{\prime}\right)^{2}}}{\mu_{1}^{\prime}}, \\
\gamma_{1}=\frac{\mu_{3}}{\left(\mu_{2}\right)^{\frac{3}{2}}}=\frac{\mu_{3}^{\prime}-3 \mu_{2}^{\prime} \mu_{1}^{\prime}+2\left(\mu_{1}^{\prime}\right)^{2}}{\left[\mu_{2}^{\prime}-\left(\mu_{1}^{\prime}\right)^{2}\right]^{\frac{3}{2}}}, \\
\gamma_{2}=\frac{\mu_{4}}{\left(\mu_{2}\right)^{2}}=\frac{\mu_{4}^{\prime}-4 \mu_{3}^{\prime} \mu_{1}^{\prime}+6 \mu_{2}^{\prime} \mu_{1}^{\prime}-3\left(\mu_{1}^{\prime}\right)^{2}}{\left[\mu_{2}^{\prime}-\left(\mu_{1}^{\prime}\right)^{2}\right]^{2}} .
\end{gathered}
$$

The moment generating function of the ETIW distribution is given by

$$
M(t)=E\left(e^{t T}\right)=\sum_{r=0}^{\infty} \frac{t^{r}}{r !} E\left(T^{r}\right) .
$$

Substituting Equation (35) into Equation (44), we have

$$
\begin{aligned}
M(t)=\nu \sum_{r=0}^{\infty} \frac{t^{r}}{r !}\left\{2 \lambda\left(\frac{1}{l+\nu+1}\right)^{\left(1-\frac{r}{\beta}-\beta\right)}-\right. & \left.(1+\lambda)\left(\frac{1}{l+v}\right)^{\left(1-\frac{r}{\beta}-\beta\right)}\right\} \sum_{k=0}^{\infty} \sum_{l=0}^{\infty}(-1)^{l} \\
& \times \frac{\Gamma(\nu)}{\Gamma(\nu-k)} \frac{\lambda^{k}}{k !}\left(\begin{array}{l}
b \\
l
\end{array}\right) \Gamma\left(1-\frac{r}{\beta}-\beta\right)
\end{aligned}
$$




\section{Reliability Analysis}

In this section, we obtained an expression for the survival analysis, hazard rate, the cumulative hazard rate, and the mean residual life function for the transmuted exponentiated inverted Weibull distribution.

\subsection{The survival function}

The transmuted exponentiated inverted Weibull distribution provides a useful tool for modelling lifetime data analysis for a given system. The survival function of ETIW distribution can be obtained from the relation given as

$$
R(t)=1-F(t)
$$

Putting Equation (6) in (42), we obtain

$$
R(t)=1-\left\{(1+\lambda) e^{-t^{-\beta}}-\lambda\left[e^{-t^{-\beta}}\right]^{2}\right\}^{\nu}
$$

The figure below illustrates the behaviour of the survival function of the ETIW distribution for some selected values of the parameters.

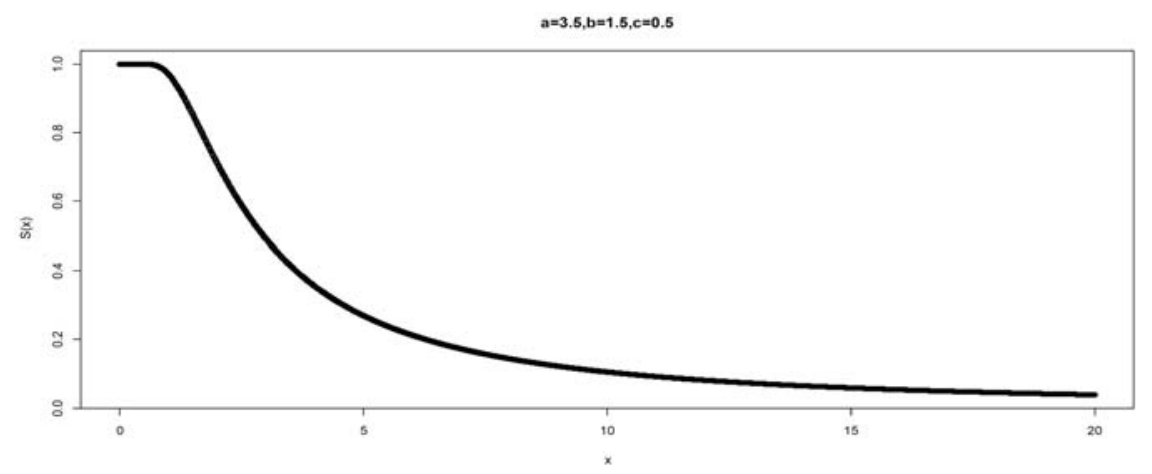

Figure 2. The graph of the survival function of ETIW distribution. 


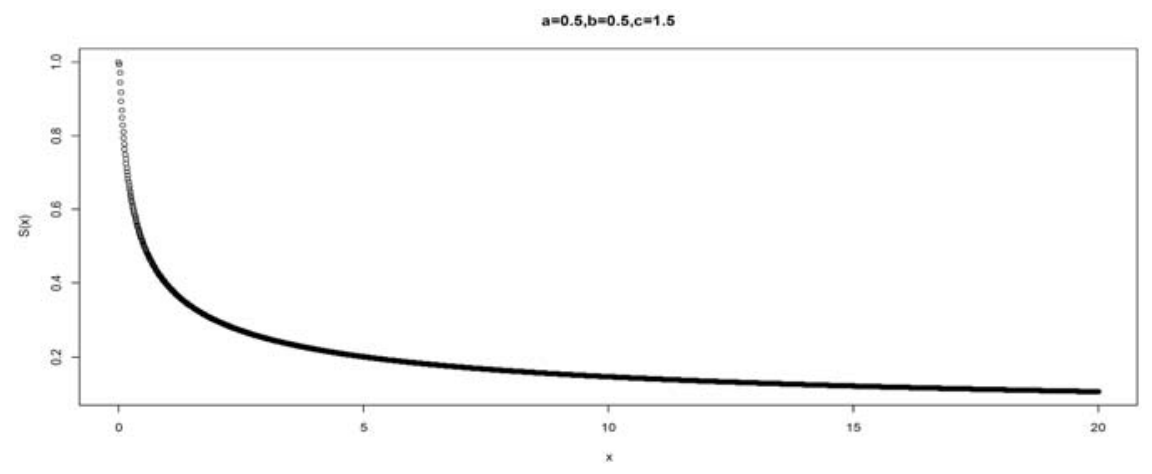

Figure 2.1. The graph of the survival function of ETIW distribution.

\subsection{The hazard rate function}

The hazard rate function is another important characteristic of interest in reliability measurement. It can be obtained by

$$
h(t)=\frac{f(t)}{R(t)} .
$$

Putting Equations (7) and (47) in (48), we have

$$
h(t)=\frac{\nu \beta x^{-\beta}\left[1+\lambda-2 \lambda e^{-t^{-\beta}}\right]\left[e^{-t^{-\beta}}\right]^{\nu}\left[1+\lambda\left(1-e^{-t^{-\beta}}\right)\right]^{\nu-1}}{1-\left\{(1+\lambda) e^{-t^{-\beta}}-\lambda\left[e^{-t^{-\beta}}\right]^{2}\right\}^{\nu}} .
$$

The graph below depicts various shape of hazard function of the ETIW distribution for various values of the parameters, this demonstrates its capability in modelling different failure phenomena. 


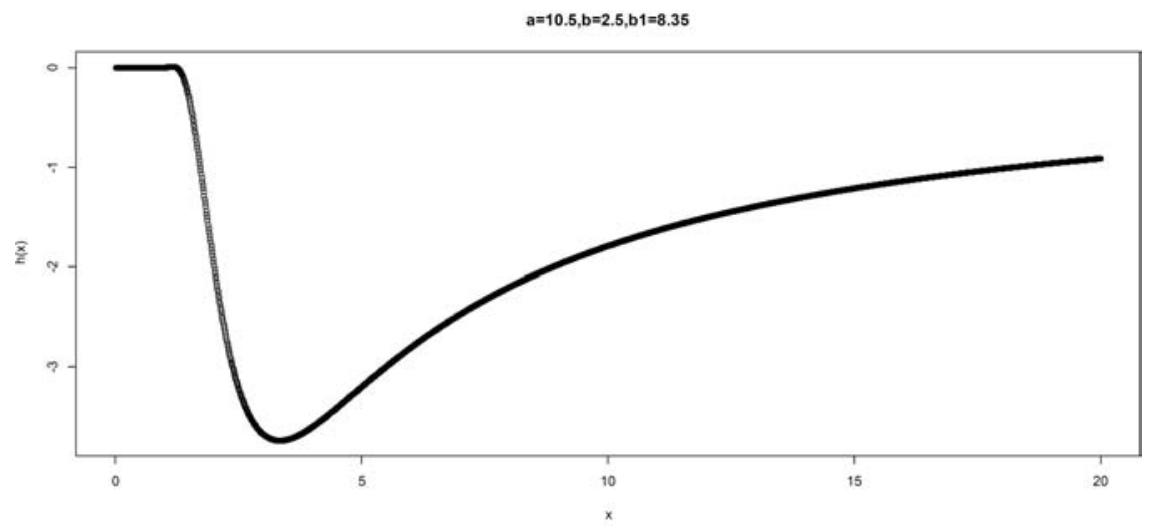

Figure 3. The graph of the hazard function of ETIW distribution.

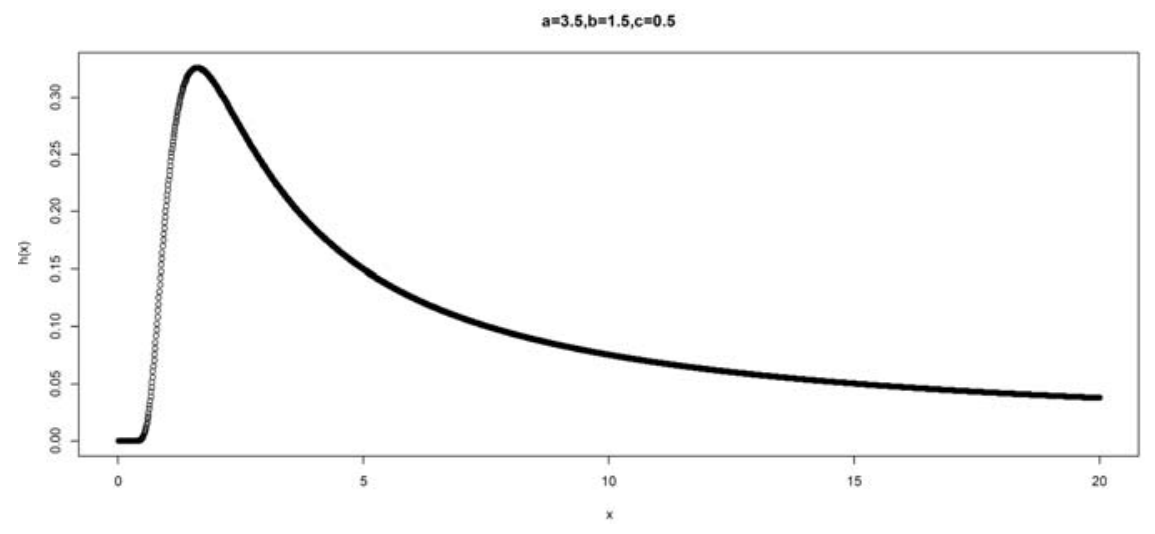

Figure 3.1. The graph of the hazard function of ETIW distribution. 


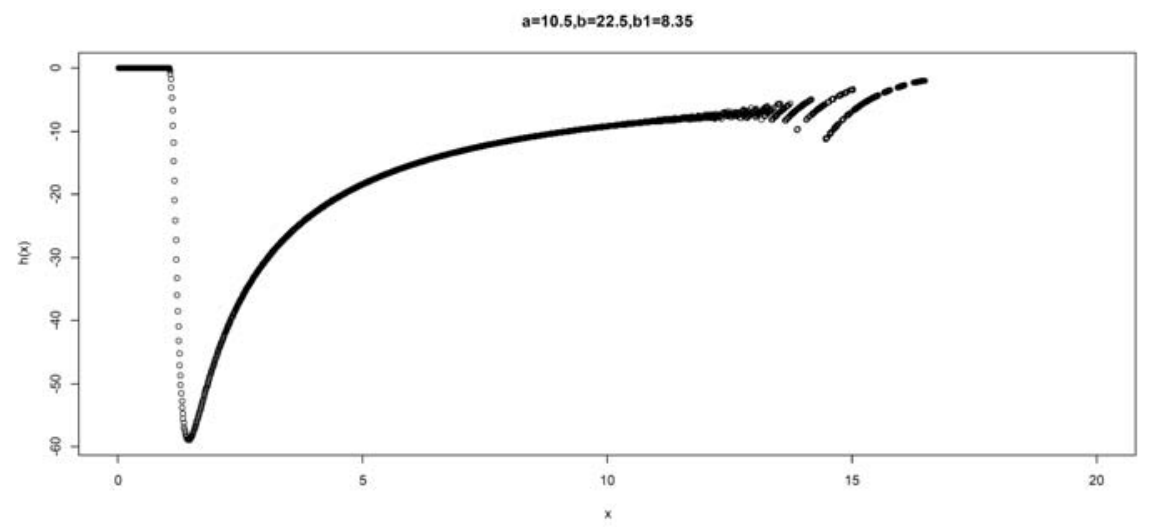

Figure 3.2. The graph of the hazard function of ETIW distribution.

\subsection{The cumulative hazard rate function}

The cumulative hazard rate function is given by

$$
H(t)=\int_{0}^{t} h(t) d t=-\ln R(t) .
$$

Inserting Equation (47) in (50), we have

$$
H(t)=-\ln \left[1-\left\{(1+\lambda) e^{-t^{-\beta}}-\lambda\left[e^{-t^{-\beta}}\right]^{2}\right\}^{\nu}\right],
$$

where $H(t)$ is the total number of failures or dearth over an interval of time, which describes how the risk of a particular system varies with time for ETIW distribution.

\section{Renyi Entropy}

The Renyi entropy of a random variable $T$ represents a measure of uncertainty. A large value of entropy indicates the greater uncertainty in the data. The Renyi [14] and Barlow et al. [13] introduced the Renyi entropy defined as 


$$
Z_{\alpha}(T)=\frac{1}{1-\alpha} \log \int_{-\infty}^{\infty} f(x)^{\alpha} d t, \quad \alpha>0 \text { and } \alpha \neq 1 .
$$

Using the pdf in (7) in Equation (52), we have

$$
Z_{\alpha}(T)=\frac{1}{1-\alpha} \log \int_{-\infty}^{\infty}\left\{\nu \beta t^{-\beta}\left[1+\lambda-2 \lambda e^{-t^{-\beta}}\right]\left[e^{-t^{-\beta}}\right]^{\nu}\left[1+\lambda\left(1-e^{-t^{-\beta}}\right)\right]^{\nu-1}\right\}^{\alpha} d t
$$

The above equation can be expressed as

$$
f(x)^{\alpha}=\sum_{l=0}^{\infty} m_{l}\left\{\beta t^{-\beta} e^{-t^{-\beta}}\right\}^{\alpha}\left\{e^{-t^{-\beta}}\right\}^{\alpha(\nu-1)+i+l}
$$

where

$$
m_{l}=\nu^{\alpha} \sum_{i=1}^{\infty}(-1)^{i+l}\left(\begin{array}{c}
\alpha(\nu-1) \\
i
\end{array}\right)\left(\begin{array}{l}
\alpha \\
l
\end{array}\right) 2^{l} \lambda^{i+k}(1+\lambda)^{\nu \alpha-i-l}
$$

The Renyi entropy is given by

$$
Z_{\alpha}(T)=\frac{1}{1-\alpha} \log \left[\sum_{l=0}^{\infty} m_{l} \int_{-\infty}^{\infty} \beta^{\alpha}\left\{t^{-\beta} e^{-t^{-\beta}}\right\}^{\alpha}\left\{e^{-t^{-\beta}}\right\}^{\alpha(\nu-1)+i+l} d t\right]
$$

Representing

$$
Q=\beta^{\alpha} \int_{-\infty}^{\infty}\left\{t^{-\beta} e^{-t^{-\beta}}\right\}^{\alpha}\left\{e^{-t^{-\beta}}\right\}^{\alpha(\nu-1)+i+l} d t
$$

Then we have

$$
Q=\beta^{\alpha} \int_{-\infty}^{\infty} t^{-\alpha \beta}\left\{e^{-(\nu \alpha+i+l) t^{-\beta}}\right\} d t
$$


We let, $w=(\nu \alpha+i+l) t^{-\beta}$, we have

$$
Q=-\left(\frac{\beta}{(\nu \alpha+i+l)}\right)^{\alpha}\left\{\frac{1}{\beta(\nu \alpha+i+l)^{-\frac{1}{\beta}}}\right\} \Gamma\left(\frac{\beta(\alpha-1)-1}{\beta}\right) .
$$

Finally, the Renyi entropy of ETIW distribution is given as

$$
Z_{\alpha}(T)=\frac{-1}{1-\alpha} \log \left[\sum_{l=0}^{\infty} m_{l}\right]\left(\frac{\beta}{(\nu \alpha+i+l)}\right)^{\alpha}\left\{\frac{1}{\beta(\nu \alpha+i+l)^{-\frac{1}{\beta}}}\right\} \Gamma\left(\frac{\beta(\alpha-1)-1}{\beta}\right) .
$$

\section{Order Statistics}

Order statistics make their appearance in many areas of statistical theory and practice. Let $T_{1}, T_{2}, \ldots, T_{n}$ be a random sample from ETIW distribution, the pdf of the $i$-th order statistics; say $T_{i: n}$ is given by

$$
f_{i: n}(t)=\frac{f(x)}{B(i, n-i+1)} \sum_{j=0}^{n-i}(-1)^{j}\left(\begin{array}{c}
n-i \\
j
\end{array}\right) F^{j+i-1}(x) .
$$

Using Equations (6) and (7) in (59), we have

$$
\begin{gathered}
f_{i: n}(t)=\frac{\nu \beta t^{-\beta}\left[1+\lambda-2 \lambda e^{-t^{-\beta}}\right]\left[e^{-t^{-\beta}}\right]^{\nu}\left[1+\lambda\left(1-e^{-t^{-\beta}}\right)\right]^{\nu-1} \sum_{j=0}^{n-i}(-1)^{j}\left(\begin{array}{c}
n-i \\
j
\end{array}\right)}{B(i, n-i+1)} \\
\times\left\{(1+\lambda) e^{-t^{-\beta}}-\lambda\left[e^{-t^{-\beta}}\right]^{2}\right\}^{\nu(j+i-1)}
\end{gathered}
$$

We can summarize the above expression as

$$
f_{i: n}(t)=\sum_{l=0}^{\infty} \phi_{1}(i, j, k, l) h_{l+1}(t)-\sum_{l=0}^{\infty} \phi_{2}(i, j, k, l) h_{l+2}(t),
$$


where

$$
\begin{aligned}
& \phi_{1}(i, j, k, l)=\frac{\nu(\lambda+1)}{B(i, n-i+1)} \sum_{j=0}^{n-i} \sum_{k=l}^{\infty} \frac{(-1)^{j+1} \lambda^{k}\left(\begin{array}{c}
n-i \\
j
\end{array}\right)\left(\begin{array}{c}
\nu(i+j)-1 \\
k
\end{array}\right)\left(\begin{array}{l}
k \\
l
\end{array}\right)}{l+1}, \\
& \phi_{2}(i, j, k, l)=\frac{2 \nu \lambda}{B(i, n-i+1)} \sum_{j=0}^{n-i} \sum_{k=l}^{\infty} \frac{(-1)^{j+1} \lambda^{k}\left(\begin{array}{c}
n-i \\
j
\end{array}\right)\left(\begin{array}{c}
\nu(i+j)-1 \\
k
\end{array}\right)\left(\begin{array}{l}
k \\
l
\end{array}\right)}{l+2} .
\end{aligned}
$$

\section{Estimation of the Parameters}

In this section, method of maximum likelihood is used to estimate the parameters and also we construct a confidence interval for the unknown parameters. Here we find the estimators for the ETIW distribution. Let $T_{1}, T_{2}, \ldots, T_{n}$ be a random sample from $T \sim \operatorname{ETIW}(\nu, \lambda, \beta)$ with observed values $t_{1}, t_{2}, \ldots, t_{n}$, then the likelihood function $L \equiv L\left(\nu, \lambda, \beta: t_{i}\right)$ can be written as

$$
L=\prod_{i=1}^{n} \nu \beta\left\{t^{-\beta}\left[1+\lambda-2 \lambda e^{-t^{-\beta}}\right]\left[e^{-t^{-\beta}}\right]^{\nu}\left[1+\lambda\left(1-e^{-t^{-\beta}}\right)\right]^{\nu-1}\right\} .
$$

Hence, the $\log$-likelihood function $l=\ln L$ becomes

$$
\begin{aligned}
\ln l=n \log (\nu)+\sum_{i=1}^{n} \log \left(\nu \beta t_{i}^{-\beta} e^{-t_{i}^{-\beta}}\right) & +\sum_{i=1}^{n} \log \left\{1+\lambda-2 \lambda e^{-t_{i}^{-\beta}}\right\} \\
& +(\nu-1) \sum_{i=1}^{n}\left[1+\lambda\left(1-e^{-t_{i}^{-\beta}}\right)\right] .
\end{aligned}
$$

The component of the score vector are obtained by differentiating (63) with respect to the parameters $\nu, \lambda$, and $\beta$ given as below:

$$
\frac{\partial l}{\partial \nu}=\frac{n}{\nu}+\sum_{i=1}^{n} \log \left(e^{-t_{i}^{-\beta}}\right)+\sum_{i=1}^{n} \log \left[1+\lambda\left(1-e^{-t_{i}^{-\beta}}\right)\right]
$$




$$
\begin{gathered}
\frac{\partial l}{\partial \lambda}=\sum_{i=1}^{n} \frac{\left\{1-2 e^{-t_{i}^{-\beta}}\right\}}{\left\{1+\lambda-2 \lambda e^{-t_{i}^{-\beta}}\right\}}+(\nu-1) \sum_{i=1}^{n} \frac{\left\{1-e^{-t_{i}^{-\beta}}\right\}}{\left\{1+\lambda\left[1-e^{-t_{i}{ }^{-\beta}}\right]\right\}}, \\
\frac{\partial l}{\partial \beta}=\sum_{i=1}^{n} \frac{\beta t_{i}^{-\beta} e^{-t_{i}^{-\beta}} \ln t_{i}\left(t_{i}^{-\beta}-1\right)}{\left\{\beta t_{i}^{-\beta} e^{-t_{i}^{-\beta}}\right\}}-2 \lambda \sum_{i=1}^{n} \frac{t_{i}^{-\beta} e^{-t_{i}^{-\beta}} \ln t_{i}}{\left\{1+\lambda-2 \lambda e^{-t_{i}^{-\beta}}\right\}} \\
+(\nu-1) \sum_{i=1}^{n} \frac{\left\{t_{i}^{-\beta} e^{-t_{i}{ }^{-\beta}} \ln t_{i}\right\}}{e^{-t_{i}^{-\beta}}}-\lambda(\nu-1) \sum_{i=1}^{n} \frac{\left\{t_{i}^{-\beta} e^{-t_{i}^{-\beta}} \ln t_{i}\right\}}{\left\{1+\lambda\left[1-e^{-t_{i}^{-\beta}}\right]\right\}} .
\end{gathered}
$$

We can derive the $(1-\delta) 100 \%$ confidence intervals of the parameters $\lambda, \nu$, and $\beta$ by using variance covariance matrix as in the following forms:

$$
\hat{\lambda} \pm Z_{\frac{\delta}{2}} \sqrt{\operatorname{var} \hat{\lambda}}, \quad \hat{\beta} \pm Z_{\frac{\delta}{2}} \sqrt{\operatorname{var} \hat{\beta}}, \quad \hat{\nu} \pm Z_{\frac{\delta}{2}} \sqrt{\operatorname{var} \hat{\nu}}
$$

where $Z_{\frac{\delta}{2}}$ is the upper $\left(\frac{\delta}{2}\right)^{\text {th }}$ percentile of the standard normal distribution.

\section{Application}

We consider a data set of the life of fatigue fracture of Kevlar $373 /$ epoxy that are subject to constant pressure at the $90 \%$ stress level until all had failed, so we have complete data with the exact times of failure. This data are: 0.0251, 0.0886, 0.0891, 0.2501, 0.3113, 0.3451, $0.4763,0.5650,0.5671,0.6566,0.6748,0.6751,0.6753,0.7696,0.8375$, $0.8391,0.8425,0.8645,0.8851,0.9113,0.9120,0.9836,1.0483,1.0596$, $1.0773,1.1733,1.2570,1.2766,1.2985,1.3211,1.3503,1.3551,1.4595$, $1.4880,1.5728,1.5733,1.7083,1.7263,1.7460,1.7630,1.7746,1.8275$, $1.8375,1.8503,1.8808,1.8878,1.8881,1.9316,1.9558,2.0048,2.0408$, $2.0903,2.1093,2.1330,2.2100,2.2460,2.2878,2.3203,2.3470,2.3513$, $2.4951,2.5260,2.9911,3.0256,3.2678,3.4045,3.4846,3.7433,3.7455$, $3.9143,4.8073,5.4005,5.4435,5.5295,6.5541$, 9.0960. For previous studies with these data sets, see Andrews and Herzberg [12]. 
Table 1. Summary of data on fatigue fracture of Kevlar 373/epoxy at $90 \%$ stress level

\begin{tabular}{|c|c|c|c|c|c|c|c|c|c|}
\hline Min & $\begin{array}{c}\text { Lower } \\
\text { quartile }\end{array}$ & median & $\begin{array}{c}\text { Upper } \\
\text { quartile }\end{array}$ & Mean & Max & Variance & Skewness & Kurtosis & Range \\
\hline 0.0251 & 0.09048 & 1.7361 & 2.2960 & 1.9590 & 9.0960 & 2.4774 & 1.9406 & 8.1608 & 9.0709 \\
\hline
\end{tabular}

Empirical density
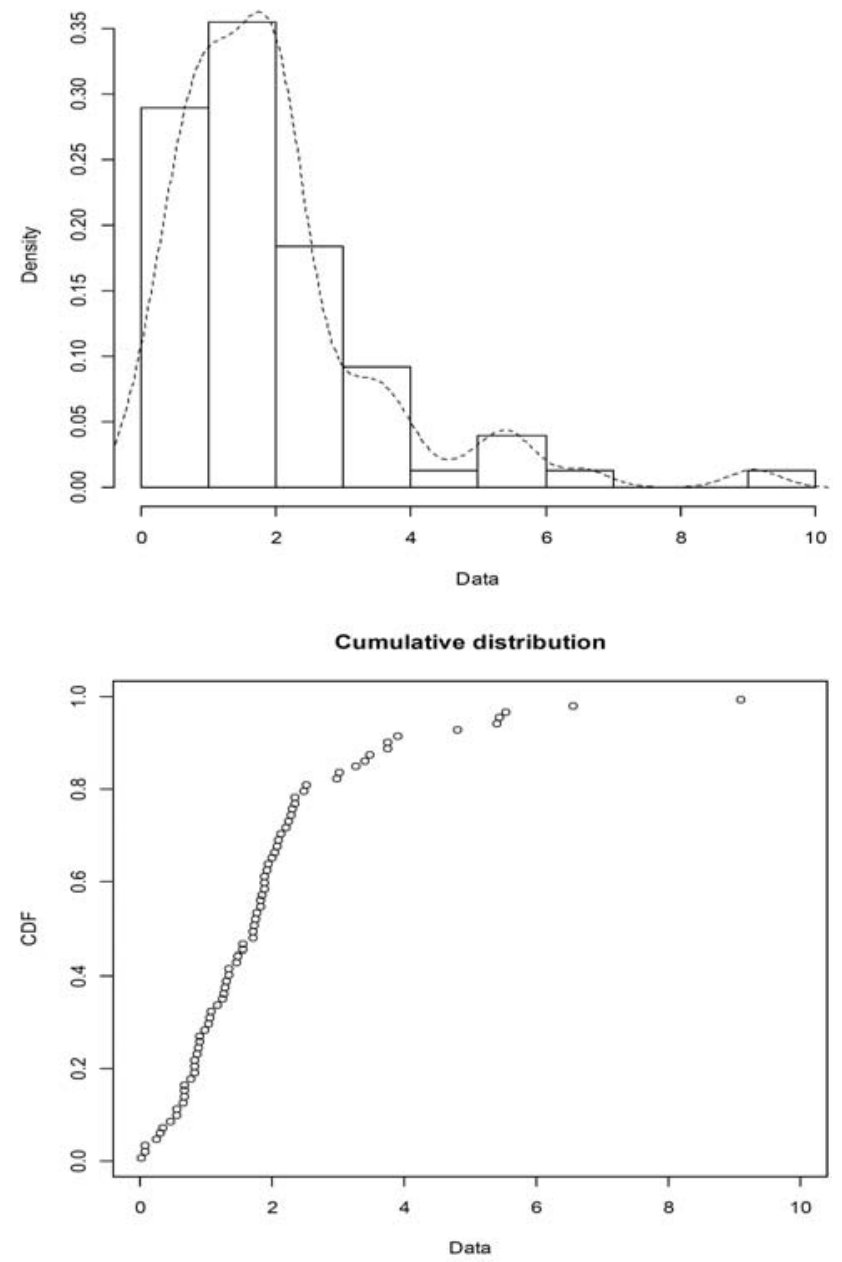

Figure 4. The graph of the empirical density and the cumulative distribution function of the Kevlar 373/epoxy data. 
Table 2. Estimated parameters of the TIWD, EIWD, and IWD

\begin{tabular}{|c|c|c|c|c|}
\hline Model & \multicolumn{3}{|c|}{ Estimates } & $l(\hat{\theta})$ \\
\hline ETIWD & 6.5763 & 0.1618 & 0.1618 & 819.463 \\
\hline$(\lambda, \beta, \nu)$ & (14.0296) & $(0.1996)$ & (1.0913) & \\
\hline TIWD & 0.7074 & 0.6903 & - & -152.483 \\
\hline$(\lambda, \beta)$ & $(0.3994)$ & $(0.0575)$ & $(-)$ & \\
\hline EIWD6.5763 & 0.8608 & 0.7588 & - & -153.539 \\
\hline$(\theta, \beta)$ & $(0.1088)$ & $(0.0541)$ & $(-)$ & \\
\hline IWD & - & 0.7322 & - & -154.278 \\
\hline$(\beta)$ & $(-)$ & $(0.0474)$ & $(-)$ & \\
\hline
\end{tabular}

Table 3. Measures of goodness of fit

\begin{tabular}{|c|c|c|c|c|c|c|c|}
\hline Model & K-S & AD & W & AIC & BIC & HQIC & CAIC \\
\hline ETIWD & 0.5086 & 2.3647 & 0.3887 & -1632.925 & -1625.933 & -1630.13 & -1632.592 \\
\hline TIWD & 0.2440 & 4.9831 & 0.8506 & 308.967 & 313.128 & 310.829 & 308.967 \\
\hline EIWD & 0.2290 & 5.9661 & 1.0351 & 311.078 & 315.740 & 312.941 & 311.243 \\
\hline IWD & 0.2291 & 5.2691 & 0.9036 & 310.556 & 312.887 & 311.487 & 310.610 \\
\hline
\end{tabular}

We employ the statistical tools for model comparison such as Kolmogorov-Smirnov (K-S) statistics, Anderson Darling (AD) statistic, Crammer von Misses statistic (W), Akaike information criterion (AIC), Consistent Akaike information criterion (CAIC), Hannan Quinine information criterion (HQIC), and Bayesian information criterion (BIC) to choose the best possible model for the data sets among the competitive models. The selection criterion is that the lowest AIC, CAIC, BIC, HQIC, $\mathrm{AD}$, and $\mathrm{W}$ statistic correspond to the best fit model.

\section{Conclusion}

Among the models considered the best model is the exponentiated transmuted inverted Weibull distribution for the two data sets. 


\section{References}

[1] A. Flair, H. Elsalloukh, E. Mendi and M. Milanova, The exponentiated inverted Weibull Distribution, Appl. Math. Inf. Sci. 6(2) (2012), 167-171.

[2] G. S. Mudholkar, D. K. Srivastava and M. Freimer, The exponentiated Weibull family: A reanalysis of the bus-motor failure data, Technometrics 37(4) (1995), 436-445.

[3] G. S. Mudholkar and A. D. Hutson, Exponentiated Weibull family: Some properties and flood data application, Commun. Statistical Theory and Method 25 (1996), 3050-3083.

[4] G. R. Aryal and Ch. P. Tsokos, Transmuted Weibull distribution: A generalization of the Weibull probability distribution, European J. Pure and Appl. Math. 4(2) (2011), 89-102.

[5] W. T. Shaw and I. R. Buckley, The alchemy ofprobability distributions: Beyond Gram-Charlier expansions and a Skew-Kurtotic-Normal distribution from a rank transmutation map, arXiv preprint arXiv:0901.0434. (2009).

[6] F. Merovci, Transmuted Rayleigh distribution, Austrian J. Stat. 42(1) (2013), 21-31.

[7] M. S. Khan and R. King, Transmuted modified Weibull distribution: A generalization of the modified Weibull probability distribution, European J. Pure and Appl. Math. 6 (2013), 66-88.

[8] S. K. Ashour and M. A. Eltehiwy, Transmuted Lomax distribution, Amer. J. Appl. Math. Stat. 1(6) (2013), 121-127.

[9] F. Merovci and L. Puka, Transmuted Pareto distribution, ProbStat Forum 7 (2014), $1-11$.

[10] I. Elbatal, L. S. Diab and N. A. Abdul-Alim, Transmuted generalized linear exponential distribution, Inter. J. Comp. Appl. 83(17) (2013), 29-37.

[11] M. S. Khan, G. R. Pasha and A. H. Pasha, Theoretical analysis of inverse Weibull distribution, WSEAS Trans. Math. 7(2) (2008).

[12] D. F. Andrews and A. M. Herzberg, Data: A collection of problems from many fields for the student and research worker, Springer Series in Statistics, New York, 1985.

[13] R. E. Barlow, R. H. Toland and T. Freeman, A Bayesian analysis of stress rupture life of Kevlar 49/epoxy (1984).

[14] A. L. Renyi, On measure on entropy and information, In Fourth Berkeley Symposium on Mathematical Statistics and Probability 1 (1961), 547-561.

[15] R. C. Gupta and R. D. Gupta, Proportional reversed hazard model and its applications, J. Stat. Plan. Infer. 137(11) (2007), 3525-3536. 
[16] A. P. Prudnikov, Y. A. Brychkov and O. I. Marichev, Integrals and Series (1, 2 and 3), Gordon and Breach Science Publishers, Amsterdan, 1986.

[17] A. A. Ogunde, O. Fatoki and O. I. Oseghale, On the application of transmuted inverted Weibull distribution, Global J. Sci. Fronttier Research, 17(6), Version 1.0

[18] Abd El Hady Ebraheim, Exponentiated Transmuted Weibull distribution A generalization of the Weibull distribution, World Academy of Science, Engineering and Technology, Inter. J. Math. Comp. Natural and Phys. Engin. 8(6) 2014. 